\title{
EXISTENCE OF FUNCTIONS IN WEIGHTED SOBOLEV SPACES
}

\author{
TOSHIHIDE FUTAMURA AND YOSHIHIRO MIZUTA
}

\author{
Dedicated to Professor Masayuki Itô \\ on the occasion of his sixtieth birthday
}

\begin{abstract}
The aim of this paper is to determine when there exists a quasicontinuous Sobolev function $u \in W^{1, p}\left(\mathbf{R}^{n} ; \mu\right)$ whose trace $\left.u\right|_{\mathbf{R}^{n-1}}$ is the characteristic function of a bounded set $E \subset \mathbf{R}^{n-1}$, where $d \mu(x)=\left|x_{n}\right|^{\alpha} d x$ with $-1<\alpha<p-1$.

As application we discuss the existence of harmonic measures for weighted $p$-Laplacians in the unit ball.
\end{abstract}

\section{$\S 1$. Introduction}

For $p>1$ and a Borel measure $\mu$, consider the relative $(p, \mu)$-capacity $\operatorname{cap}_{p, \mu}(\cdot ; \Omega)$ for an open set $\Omega \subset \mathbf{R}^{n}$. For a compact set $K \subset \Omega$, it is defined by

$$
\operatorname{cap}_{p, \mu}(K ; \Omega)=\inf \int_{\Omega}|\nabla u|^{p} d \mu
$$

where the infimum is taken over all functions $u \in C_{c}^{\infty}(\Omega)$ such that $u \geq 1$ on $K$; here $C_{c}^{\infty}(\Omega)$ denotes the space of infinitely differentiable functions with compact support in $\Omega$. We extend the capacity $\operatorname{cap}_{p, \mu}(\cdot ; \Omega)$ in the usual way (see Section 2).

For a subset $E$ of $\mathbf{R}^{n}$, denote the characteristic function of $E$ by $\chi_{E}$. We use the notation $W_{E}^{1, p}\left(\mathbf{R}^{n} ; \mu\right)$ to denote the class of $(p, \mu)$-quasicontinuous Sobolev functions $u \in W^{1, p}\left(\mathbf{R}^{n} ; \mu\right)$ such that $\left.u\right|_{\mathbf{R}^{n-1}}$ is equal to $\chi_{E}(p, \mu)$ q.e. on $\mathbf{R}^{n-1}$; here we identify $\mathbf{R}^{n-1}$ with the hyperplane $\mathbf{R}^{n-1} \times\{0\}$. Throughout this paper, for $-1<\alpha<p-1$ we consider the Borel measure

$$
d \mu(x)=\left|x_{n}\right|^{\alpha} d x
$$

Received March 23, 2000.

2000 Mathematics Subject Classification: Primary 31B30. 
where $x=\left(x_{1}, \ldots, x_{n-1}, x_{n}\right) \in \mathbf{R}^{n}$ and $d x$ denotes the usual Lebesgue measure.

Motivated by the work of Nakai on the existence of Dirichlet finite harmonic measures (cf. $[10,11,12]$ ), we consider the problem to determine when $W_{E}^{1, p}\left(\mathbf{R}^{n} ; \mu\right) \neq \emptyset$. Our aim in this paper is to show the following result, as a generalization of Herron-Koskela [4] which treats the case $\alpha=0$.

TheOREM 1.1. Let $E \subset \mathbf{R}^{n-1}$ be a bounded Borel set, $1<p<\infty$ and $-1<\alpha<p-1$. Suppose $W_{E}^{1, p}\left(\mathbf{R}^{n} ; \mu\right) \neq \emptyset$.

(a) If $p>n+\alpha$, then $E=\emptyset$.

(b) If $2+\alpha \leq p \leq n+\alpha$, then $\mathcal{H}^{n-1}(E)=0$.

(c) If $\mathcal{H}^{n-1}(E)=0$, then $\operatorname{cap}_{p, \mu}(E)=0$.

Here $\mathcal{H}^{s}$ denotes the $s$-dimensional Hausdorff measure.

In case $p \geq 2+\alpha, E$ has $(p, \mu)$-capacity zero if $W_{E}^{1, p}\left(\mathbf{R}^{n} ; \mu\right) \neq \emptyset$. In Section 5, we give some examples of bounded Borel sets $E \subset \mathbf{R}^{n-1}$ with $\mathcal{H}^{n-1}(E)>0$ and $W_{E}^{1, p}\left(\mathbf{R}^{n} ; \mu\right) \neq \emptyset$ for $1<p<2+\alpha$.

As applications of our results, we discuss the existence of harmonic measures for the weighted $p$-Laplace equation

$$
-\operatorname{div}\left(\omega(x)|\nabla u(x)|^{p-2} \nabla u(x)\right)=0
$$

in the unit ball $\mathbf{B}$ in $\mathbf{R}^{n}$. In this paper, we consider

$$
\omega(x)=|1-| x||^{\alpha} \quad(-1<\alpha<p-1)
$$

as the weight function, which is in the Muckenhoupt $A_{p}$ class. Further, letting $d \nu(x)=\omega(x) d x$, we say that a function $w$ is $(p, \nu)$-Dirichlet finite if

$$
\int_{\mathbf{B}}|\nabla w(x)|^{p} d \nu(x)<\infty .
$$

A $(p, \nu)$-Dirichlet finite function $u$ on $\mathbf{B}$ is said to be $(p, \nu)$-harmonic if it is a continuous weak solution of (1.1) in $\mathbf{B}$. We say that $w$ is a $(p, \nu)$ harmonic measure on $\mathbf{B}$ if $w$ is $(p, \nu)$-harmonic in $\mathbf{B}$ and the greatest $(p, \nu)$ harmonic minorant of $\min \{w, 1-w\}$ is zero. We see that $0 \leq w \leq 1$ if $w$ is a $(p, \nu)$-harmonic measure. For elementary properties of $(p, \nu)$-harmonic functions and $(p, \nu)$-harmonic measures, see Heinonen-Kilpeläinen-Martio [3] and Nakai $[10,11,12]$. 
Corollary 1.1. Let $1<p<\infty$ and $-1<\alpha<p-1$.

(1) If $p \geq 2+\alpha$, then every $(p, \nu)$-Dirichlet finite $(p, \nu)$-harmonic measure on $\mathbf{B}$ is constant.

(2) For each $1<p<2+\alpha$, there exists a non-constant $(p, \nu)$-Dirichlet finite $(p, \nu)$-harmonic measure on $\mathbf{B}$.

\section{$\S 2 . \quad$ Preliminaries}

Let $\mathbf{R}^{n}$ be the $n$-dimensional Euclidean space $(n \geq 2)$. For a point $x \in \mathbf{R}^{n}$, we write $x=\left(x^{\prime}, x_{n}\right)$, where $x^{\prime}=\left(x_{1}, x_{2}, \ldots, x_{n-1}\right) \in \mathbf{R}^{n-1}$. Let $B(x, r)$ denote the open ball centered at $x$ with radius $r$, and $\mathcal{H}^{s}$ the $s$-dimensional Hausdorff measure. For $1<p<\infty$, let $W^{1, p}\left(\mathbf{R}^{n} ; \mu\right)$ denote the Sobolev space of all functions $u \in L^{p}\left(\mathbf{R}^{n} ; \mu\right)$ whose gradient, denoted by $\nabla u$, belongs to $L^{p}\left(\mathbf{R}^{n} ; \mu\right)$, where $d \mu(x)=\left|x_{n}\right|^{\alpha} d x$ with $-1<\alpha<p-1$ as above.

Suppose that $\Omega \subset \mathbf{R}^{n}$ is open. For a compact subset $K$ of $\Omega$, let

$$
W(K, \Omega)=\left\{u \in C_{c}^{\infty}(\Omega): u \geq 1 \text { on } K\right\}
$$

and define

$$
\operatorname{cap}_{p, \mu}(K ; \Omega)=\inf _{u \in W(K, \Omega)} \int_{\Omega}|\nabla u|^{p} d \mu
$$

Further, we set

$$
\operatorname{cap}_{p, \mu}(U ; \Omega)=\sup _{\substack{K \subset U \\ K: \text { compact }}} \operatorname{cap}_{p, \mu}(K ; \Omega)
$$

for an open set $U \subset \Omega$, and then

$$
\operatorname{cap}_{p, \mu}(E ; \Omega)=\inf _{\substack{E \subset U \subset \Omega \\ U: \text { open }}} \operatorname{cap}_{p, \mu}(U ; \Omega)
$$

for an arbitrary set $E \subset \Omega$.

The number $\operatorname{cap}_{p, \mu}(E ; \Omega)$ is called the (variational) $(p, \mu)$-capacity of $E$ relative to $\Omega$. We know that $\operatorname{cap}_{p, \mu}(E ; \Omega)$ is an outer Choquet capacity (see $[2,3,7])$. We say that $E \subset \mathbf{R}^{n}$ has $(p, \mu)$-capacity zero, denoted by $\operatorname{cap}_{p, \mu}(E)=0$, if $\operatorname{cap}_{p, \mu}(E \cap \Omega ; \Omega)=0$ for all open sets $\Omega \subset \mathbf{R}^{n}$.

We say that a property holds $(p, \mu)$-quasieverywhere, often abbreviated to $(p, \mu)$-q.e., if it holds except on a set of $(p, \mu)$-capacity zero. A function $u$ on a bounded open set $\Omega$ is said to be $(p, \mu)$-quasicontinuous if given 
$\varepsilon>0$ there exists an open set $E \subset \Omega$ such that $\operatorname{cap}_{p, \mu}(E ; \Omega)<\varepsilon$ and $u$ is continuous as a function on $\Omega \backslash E$. Further we say that a function $u$ is $(p, \mu)$ quasicontinuous in $\mathbf{R}^{n}$ if $u$ is $(p, \mu)$-quasicontinuous in $\Omega$ for all bounded open set $\Omega$.

The following lemma can be obtained by an elementary calculation and our assumption on $\alpha$.

Lemma 2.1. Let $x=\left(x^{\prime}, 0\right) \in \mathbf{R}^{n}$ and $r>0$. Then

$$
\mu(B(x, r))=c_{1} r^{n+\alpha},
$$

where $c_{1}=\mu(B(0,1))<\infty$.

Lemma 2.2. Let $x=\left(x^{\prime}, 0\right) \in \mathbf{R}^{n}$ and $r>0$. Then

$$
\operatorname{cap}_{p, \mu}(B(x, r) ; B(x, 2 r))=c_{2} r^{n+\alpha-p},
$$

where $c_{2}=\operatorname{cap}_{p, \mu}(B(0,1) ; B(0,2))<\infty$.

Proof. For $a>0$, let $u \in W(\bar{B}(0, a), B(0,2 a))$ where $\bar{B}(x, r)$ denotes the closure of $B(x, r)$. If we set $v(y)=u((y-x) / r)$ for $r>0$, then $v \in$ $W(\bar{B}(x, r a), B(x, 2 r a))$ and it follows from the definition of the capacity that

$$
\begin{aligned}
\operatorname{cap}_{p, \mu}(\bar{B}(x, r a) ; B(x, 2 r a)) & \leq \int_{B(x, 2 r a)}|\nabla v(y)|^{p} d \mu(y) \\
& =r^{-p+n+\alpha} \int_{B(0,2 a)}|\nabla u(y)|^{p} d \mu(y),
\end{aligned}
$$

which gives

$$
\operatorname{cap}_{p, \mu}(\bar{B}(x, r a) ; B(x, 2 r a)) \leq c(a) r^{n+\alpha-p},
$$

where $c(a)=\operatorname{cap}_{p, \mu}(\bar{B}(0, a) ; B(0,2 a))$. Hence we obtain

$$
c(a) \leq c(r a) r^{-(n+\alpha-p)} \leq c(a)
$$

and thus

$$
\operatorname{cap}_{p, \mu}(\bar{B}(x, r) ; B(x, 2 r))=c_{2} r^{n+\alpha-p},
$$

where $c_{2}=c(1)<\infty$, by Lemma 2.1. It is also clear from the above equality that

$$
\operatorname{cap}_{p, \mu}(B(x, r) ; B(x, 2 r))=\operatorname{cap}_{p, \mu}(\bar{B}(x, r) ; B(x, 2 r))
$$


In the same way as in [5, Theorem 22], we have the following result (see also [3, Theorem 2.32]).

Corollary 2.1. If $E \subset \mathbf{R}^{n-1}$ and $\operatorname{cap}_{p, \mu}(E)=0$, then $E$ has Hausdorff dimension at most $n-p+\alpha$.

Lemma 2.3. (cf. [3, Theorems 4.4, 4.12] and [7])

(1) For each $u \in W^{1, p}\left(\mathbf{R}^{n} ; \mu\right)$, there exists a $(p, \mu)$-quasicontinuous representative $u^{*}$ which is equal to $u$ a.e. on $\mathbf{R}^{n}$.

(2) If $u^{*}$ and $v^{*}$ are $(p, \mu)$-quasicontinuous and $u^{*}=v^{*}$ a.e. on $\mathbf{R}^{n}$, then $u^{*}=v^{*}(p, \mu)$-q.e. on $\mathbf{R}^{n}$.

A set $A$ is called $(p, \mu)$-thin at a point $x$ if

$$
\int_{0}^{1}\left(\frac{\operatorname{cap}_{p, \mu}(A \cap B(x, r) ; B(x, 2 r))}{\operatorname{cap}_{p, \mu}(B(x, r) ; B(x, 2 r))}\right)^{1 /(p-1)} r^{-1} d r<\infty
$$

$A$ is $(p, \mu)$-thick at $x$ if $A$ is not $(p, \mu)$-thin at $x$. We say that a function $u$ is $(p, \mu)$-finely continuous at $x$ if there exists a set $A$ which is $(p, \mu)$-thin at $x$ such that

$$
u(x)=\lim _{\substack{y \rightarrow x \\ y \notin A}} u(y) .
$$

Lemma 2.4. (cf. Meyers [6] and [3, Section 12]) Let $u \in W^{1, p}\left(\mathbf{R}^{n} ; \mu\right)$. If $u$ is $(p, \mu)$-quasicontinuous on $\mathbf{R}^{n}$, then it is $(p, \mu)$-finely continuous $(p, \mu)$ q.e. on $\mathbf{R}^{n}$.

Lemma 2.5. (cf. [3, Section 12] and [9]) Let $p>n+\alpha$.

(1) $E \subset \mathbf{R}^{n-1}$ has capacity zero if and only if $E$ is empty.

(2) If $u \in W^{1, p}\left(\mathbf{R}^{n} ; \mu\right)$ is $(p, \mu)$-quasicontinuous on $\mathbf{R}^{n}$, then $\left.u\right|_{\mathbf{R}^{n-1}}$ is continuous on $\mathbf{R}^{n-1}$.

Proof. In this proof, we identify $x^{\prime} \in \mathbf{R}^{n-1}$ with $\left(x^{\prime}, 0\right) \in \mathbf{R}^{n-1} \times \mathbf{R}^{1}$. First we show that if $x^{\prime} \in \mathbf{R}^{n-1} \cap B(0, N), N>0$, then

$$
\int_{B(0, N)}\left|x^{\prime}-y\right|^{1-n} f(y) d y \leq C N^{(p-n-\alpha) / p}\|f\|_{L^{p}\left(\mathbf{R}^{n} ; \mu\right)}
$$


for every nonnegative measurable function $f$ on $\mathbf{R}^{n}$. In fact, we have by Hölder's inequality

$$
\begin{aligned}
& \int_{B(0, N)}\left|x^{\prime}-y\right|^{1-n} f(y) d y \\
\leq & \left(\int_{B(0, N)}\left|x^{\prime}-y\right|^{(1-n) p^{\prime}}\left|y_{n}\right|^{-\alpha p^{\prime} / p} d y\right)^{1 / p^{\prime}}\left(\int_{B(0, N)} f(y)^{p}\left|y_{n}\right|^{\alpha} d y\right)^{1 / p} \\
\leq & C\left(N+\left|x^{\prime}\right|\right)^{(p-n-\alpha) / p}\|f\|_{L^{p}\left(\mathbf{R}^{n} ; \mu\right)}
\end{aligned}
$$

where $1 / p+1 / p^{\prime}=1$.

In view of (2.1), we see easily that

$$
\operatorname{cap}_{p, \mu}\left(\left\{x^{\prime}\right\} ; B(0, N)\right) \geq C N^{-(p-n-\alpha)},
$$

so that $\operatorname{cap}_{p, \mu}\left(\left\{x^{\prime}\right\}\right)>0$, which proves $(1)$.

As another application of (2.1), we show that the potential

$$
u\left(x^{\prime}\right)=\int_{B(0, N)}\left|x^{\prime}-y\right|^{1-n} f(y) d y \quad\left(x^{\prime} \in \mathbf{R}^{n-1}\right)
$$

is continuous on $\mathbf{R}^{n-1} \cap B(0, N)$ for every $f \in L^{p}\left(\mathbf{R}^{n} ; \mu\right)$. To show this, we note that if $z^{\prime} \in \mathbf{R}^{n-1} \cap B(0, N)$ and $0<s<N-\left|z^{\prime}\right|$, then

$$
\int_{B(0, N)-B\left(z^{\prime}, s\right)}\left|x^{\prime}-y\right|^{1-n} f(y) d y
$$

is continuous at $z^{\prime}$. Further, in view of (2.1), if $x^{\prime} \in \mathbf{R}^{n-1} \cap B\left(z^{\prime}, s\right)$, then

$$
\int_{B\left(z^{\prime}, s\right)}\left|x^{\prime}-y\right|^{1-n} f(y) d y \leq \int_{B\left(x^{\prime}, 2 s\right)}\left|x^{\prime}-y\right|^{1-n} f(y) d y
$$

is small enough when $s$ is small. Hence $u\left(x^{\prime}\right)$ is continuous at $z^{\prime}$. With the aid of Sobolev's integral representation of $u \in W^{1, p}\left(\mathbf{R}^{n} ; \mu\right)$ (cf. [9]), we see that $\left.u\right|_{\mathbf{R}^{n-1}}$ is continuous, so that (2) follows.

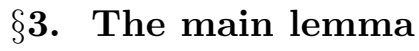

Here we prepare the following technical lemma needed for the proof of Theorem 1.1. 
LEMmA 3.1. Let $\Omega$ be a bounded open set in $\mathbf{R}^{n}$. If $A$ is a Borel set with its closure in $\Omega$ and $\mathcal{H}^{n}(A)>0$, then

$$
\iint_{A \times(\Omega \backslash A)}|x-y|^{-n-1} d x d y=\infty .
$$

Proof. Set

$$
\tilde{A}=\left\{x \in A: \lim _{r \rightarrow 0} \frac{\mathcal{H}^{n}(A \cap B(x, r))}{\mathcal{H}^{n}(B(x, r))}=1\right\} .
$$

Then $\mathcal{H}^{n}(A \backslash \tilde{A})=0$ (cf. [2]), so that we may assume that $A=\tilde{A}$, that is, every point of $A$ is the Lebesgue point of $A$. Further we may assume without loss of generality that the origin $0 \in A$. By our assumption, there exists $r_{0}>0$ such that $\mathcal{H}^{n}\left(A \backslash B\left(0, r_{0}\right)\right)>0$ and $B(x, r) \subset \Omega$ whenever $B(x, r) \cap A \neq \emptyset$ and $0<r<r_{0}$. Set $P(x)=\left\{t x: t \geq r_{0}\right\}$ for each $x \in \mathbf{R}^{n}$ and $S=\left\{x \in \mathbf{R}^{n}:|x|=1\right.$ and $\left.P(x) \cap A \neq \emptyset\right\}$. Then we see from Fubini's theorem that $\mathcal{H}^{n-1}(S)>0$.

Take $r_{1}$ such that $0<r_{1}<r_{0}$. By the intermediate value theorem, for each $x \in S$ we can find $B\left(x^{(1)}, r_{x}^{(1)}\right)$ such that $x^{(1)} \in P(x), 0<r_{x}^{(1)}<r_{1}$ and

$$
\frac{\mathcal{H}^{n}\left(A \cap B\left(x^{(1)}, r_{x}^{(1)}\right)\right)}{\mathcal{H}^{n}\left(B\left(x^{(1)}, r_{x}^{(1)}\right)\right)}=\frac{3}{4}
$$

Now we denote by $B^{*}(x, r)$ the projection of $B(x, r)$ to $\partial B(0,1)$. By the covering lemma, we can find a countable family $\left\{B_{1 j}\right\}$, where $B_{1 j}=B\left(z_{j}^{(1)}, r_{j}^{(1)}\right)$, such that $\left|z_{j}^{(1)}\right| \geq r_{0}, r_{j}^{(1)}<r_{1},\left\{B_{1 j}^{*}\right\}$ is disjoint and

$$
\bigcup_{x \in S} B^{*}\left(x^{(1)}, r_{x}^{(1)}\right) \subset \bigcup_{j} B^{*}\left(z_{j}^{(1)}, 5 r_{j}^{(1)}\right) .
$$

Since $\mathcal{H}^{n-1}\left(B^{*}(x, r)\right) \leq c_{3} r^{n-1}$ for $|x| \geq r_{0}$ with a constant $c_{3}>0$, depending only on $r_{0}$, we have

$$
\begin{aligned}
c_{3} \sum_{j}\left(5 r_{j}^{(1)}\right)^{n-1} & \geq \sum_{j} \mathcal{H}^{n-1}\left(B^{*}\left(z_{j}^{(1)}, 5 r_{j}^{(1)}\right)\right) \\
& \geq \mathcal{H}^{n-1}\left(\bigcup_{x \in S} B^{*}\left(x^{(1)}, r_{x}^{(1)}\right)\right) \\
& \geq \mathcal{H}^{n-1}(S) .
\end{aligned}
$$


Hence we can find a positive integer $N_{1}$ such that

$$
\sum_{j=1}^{N_{1}}\left(r_{j}^{(1)}\right)^{n-1} \geq c_{4}
$$

where $c_{4}=\left(2 c_{3} 5^{n-1}\right)^{-1} \mathcal{H}^{n-1}(S)>0$. Since $\left\{B_{1 j}\right\}_{j=1}^{N_{1}}$ is disjoint, we have

$$
\begin{aligned}
& \iint_{A \times(\Omega \backslash A)}|x-y|^{-n-1} d x d y \\
\geq & \int_{\left(\bigcup_{j=1}^{N_{1}} B_{1 j}\right) \cap A}\left(\int_{\Omega \backslash A}|x-y|^{-n-1} d x\right) d y \\
\geq & \sum_{j=1}^{N_{1}} \int_{B_{1 j} \cap A}\left(\int_{B_{1 j} \backslash A}|x-y|^{-n-1} d x\right) d y \\
\geq & \sum_{j=1}^{N_{1}}\left(2 r_{j}^{(1)}\right)^{-n-1} \mathcal{H}^{n}\left(B_{1 j} \backslash A\right) \mathcal{H}^{n}\left(B_{1 j} \cap A\right) \\
= & \sum_{j=1}^{N_{1}}\left(2 r_{j}^{(1)}\right)^{-n-1} \frac{1}{4} \mathcal{H}^{n}\left(B_{1 j}\right) \frac{3}{4} \mathcal{H}^{n}\left(B_{1 j}\right) \\
> & 2^{-n-4} \sigma_{n}^{2} \sum_{j=1}^{N_{1}}\left(r_{j}^{(1)}\right)^{n-1}>c_{5},
\end{aligned}
$$

in view of $(3.1)$, where $c_{5}=2^{-n-5} \sigma_{n}^{2} c_{4}$. The above inequalities also imply that

$$
\sum_{j=1}^{N_{1}} \int_{\left(B_{1 j} \cap A\right) \backslash G}\left(\int_{B_{1 j} \backslash A}|x-y|^{-n-1} d x\right) d y \geq c_{5}
$$

whenever $G \subset \mathbf{R}^{n}$ satisfies $\mathcal{H}^{n}\left(B_{1 j} \cap A \backslash G\right) \geq \mathcal{H}^{n}\left(B_{1 j}\right) / 2$ for $1 \leq j \leq N_{1}$.

For $\gamma_{1}=\min _{1 \leq j \leq N_{1}} r_{j}^{(1)}$ and $\varepsilon_{1}>0$, take $r_{2}$ such that $0<r_{2}<\varepsilon_{1} \gamma_{1}$. Next, for each $x \in S$, find $B\left(x^{(2)}, r_{x}^{(2)}\right)$ such that $x^{(2)} \in P(x), 0<r_{x}^{(2)}<r_{2}$ and

$$
\frac{\mathcal{H}^{n}\left(A \cap B\left(x^{(2)}, r_{x}^{(2)}\right)\right)}{\mathcal{H}^{n}\left(B\left(x^{(2)}, r_{x}^{(2)}\right)\right)}=\frac{3}{4}
$$


By the same considerations as above, we can take a family $\left\{B_{2 j}\right\}_{j=1}^{N_{2}}$, where $B_{2 j}=B\left(z_{j}^{(2)}, r_{j}^{(2)}\right)$, such that $\left|z_{j}^{(2)}\right| \geq r_{0}, r_{j}^{(2)}<r_{2},\left\{B_{2 j}^{*}\right\}_{j=1}^{N_{2}}$ is disjoint and

$$
\sum_{j=1}^{N_{2}} \int_{\left(B_{2 j} \cap A\right) \backslash G}\left(\int_{B_{2 j} \backslash A}|x-y|^{-n-1} d x\right) d y \geq c_{5}
$$

whenever $G \subset \mathbf{R}^{n}$ satisfies $\mathcal{H}^{n}\left(B_{2 j} \cap A \backslash G\right) \geq \mathcal{H}^{n}\left(B_{2 j}\right) / 2$ for $1 \leq j \leq N_{2}$. Since $\left\{B_{2 j}^{*}\right\}_{j=1}^{N_{2}}$ is disjoint, we see from Fubini's theorem that

$$
\mathcal{H}^{n}\left(B_{1 j_{1}} \cap \bigcup_{j_{2}=1}^{N_{2}} B_{2 j_{2}}\right) \leq\left(\max _{j_{2}} 2 r_{j_{2}}^{(2)}\right) c_{6} \mathcal{H}^{n-1}\left(B_{1 j_{1}}^{*}\right) \leq 2 c_{3} c_{6}\left(r_{j_{1}}^{(1)}\right)^{n-1} r_{2}
$$

so that

$$
\begin{aligned}
\mathcal{H}^{n}\left(B_{1 j_{1}} \cap A \backslash \bigcup_{j_{2}=1}^{N_{2}} B_{2 j_{2}}\right) & \geq \mathcal{H}^{n}\left(B_{1 j_{1}} \cap A\right)-\mathcal{H}^{n}\left(B_{1 j_{1}} \cap \bigcup_{j_{2}=1}^{N_{2}} B_{2 j_{2}}\right) \\
& \geq 3 \mathcal{H}^{n}\left(B_{1 j_{1}}\right) / 4-2 c_{3} c_{6}\left(r_{j_{1}}^{(1)}\right)^{n-1} r_{2} \\
& \geq\left(3 / 4-c_{7} \varepsilon_{1}\right) \mathcal{H}^{n}\left(B_{1 j_{1}}\right),
\end{aligned}
$$

where $c_{6}$ and $c_{7}$ are positive constants. Taking $\varepsilon_{1}=2^{-3} / c_{7}$, we have by (3.2) and (3.3)

$$
\begin{aligned}
& \iint_{A \times(\Omega \backslash A)}|x-y|^{-n-1} d x d y \\
& \geq \int_{\bigcup_{j_{1}=1}^{N_{1}} B_{1 j_{1}} \cap A \backslash \bigcup_{j_{2}=1}^{N_{2}} B_{2 j_{2}}}\left(\int_{\Omega \backslash A}|x-y|^{-n-1} d x\right) d y \\
&+\int_{\bigcup_{j_{2}=1}^{N_{2}} B_{2 j_{2}} \cap A}\left(\int_{\Omega \backslash A}|x-y|^{-n-1} d x\right) d y \geq 2 c_{5} .
\end{aligned}
$$

Setting $\varepsilon_{i}=2^{-i-2} / c_{7}$ for each nonnegative integer $i$, we apply the above arguments repeatedly, and obtain $\left\{B_{i j}\right\}_{j=1}^{N_{i}}$, where $B_{i j}=B\left(z_{j}^{(i)}, r_{j}^{(i)}\right)$, such that $\left\{B_{i j}^{*}\right\}_{j=1}^{N_{i}}$ is disjoint, $\left|z_{j}^{(i)}\right| \geq r_{0}, r_{j}^{(i)}<\varepsilon_{i-1} \min \left\{r_{j}^{(i-1)}: 1 \leq j \leq N_{i-1}\right\}$, $\mathcal{H}^{n}\left(A \cap B_{i j}\right)=3 \mathcal{H}^{n}\left(B_{i j}\right) / 4$ and

$$
\sum_{j=1}^{N_{i}} \int_{B_{i j} \cap A \backslash G}\left(\int_{B_{i j} \backslash A}|x-y|^{-n-1} d x\right) d y \geq c_{5}
$$


whenever $G \subset \mathbf{R}^{n}$ satisfies $\mathcal{H}^{n}\left(B_{i j} \cap A \backslash G\right) \geq \mathcal{H}^{n}\left(B_{i j}\right) / 2$ for $1 \leq j \leq N_{i}$. For $1 \leq i \leq k-1$ and $1 \leq j_{i} \leq N_{i}$, we have

$$
\mathcal{H}^{n}\left(B_{i j_{i}} \cap A \backslash G_{i}\right) \geq\left(3 / 4-c_{7} \sum_{l=i}^{k-1} \varepsilon_{l}\right) \mathcal{H}^{n}\left(B_{i j_{i}}\right) \geq \mathcal{H}^{n}\left(B_{i j_{i}}\right) / 2
$$

where $G_{i}=\cup_{l=i+1}^{k} \cup_{j_{l}=1}^{N_{l}} B_{l j_{l}}$. Thus we have by $(3.4)$

$$
\begin{aligned}
& \iint_{A \times(\Omega \backslash A)}|x-y|^{-n-1} d x d y \\
\geq & \sum_{i=1}^{k} \int_{\bigcup_{j_{i}=1}^{N_{i}} B_{i j_{i}} \cap A \backslash G_{i}}\left(\int_{\Omega \backslash A}|x-y|^{-n-1} d x\right) d y \\
\geq & \sum_{i=1}^{k} \sum_{j_{i}=1}^{N_{i}} \int_{B_{i j_{i}} \cap A \backslash G_{i}}\left(\int_{B_{i j} \backslash A}|x-y|^{-n-1} d x\right) d y \\
\geq & k c_{5},
\end{aligned}
$$

which proves the present lemma by the arbitrariness of $k$.

\section{§4. Proof of Theorem 1.1}

We are now ready to prove Theorem 1.1.

Proof of (a). In case $p>n+\alpha$, Lemma 2.5 shows that $W_{E}^{1, p}\left(\mathbf{R}^{n} ; \mu\right)$ is empty for every bounded nonempty set $E \subset \mathbf{R}^{n-1}$.

Proof of (b). Assume that $2+\alpha \leq p \leq n+\alpha$ and $W_{E}^{1, p}\left(\mathbf{R}^{n} ; \mu\right) \neq \emptyset$. If $u \in W^{1, p}\left(\mathbf{R}^{n} ; \mu\right)$ is $(p, \mu)$-quasicontinuous on $\mathbf{R}^{n}$, then, in view of $[9,13]$

$$
\iint_{\Omega \times \Omega} \frac{\left|u\left(x^{\prime}, 0\right)-u\left(y^{\prime}, 0\right)\right|^{p}}{\left|x^{\prime}-y^{\prime}\right|^{n+p-(2+\alpha)}} d x^{\prime} d y^{\prime}<\infty
$$

whenever $\Omega$ is a bounded open set in $\mathbf{R}^{n-1}$, so that

$$
\iint_{E \times(\Omega \backslash E)}\left|x^{\prime}-y^{\prime}\right|^{-n-p+2+\alpha} d x^{\prime} d y^{\prime}<\infty,
$$

which together with Lemma 3.1 implies that $E$ is of measure zero. Thus (b) holds. 
Proof of (c). We claim that if $\mathcal{H}^{n-1}(E)=0$, then $F=\mathbf{R}^{n-1} \backslash E$ is $(p, \mu)$-thick at each point of $E$. Fix $x \in E$ and $r>0$. Let $K \subset F \cap B(x, r)$ be compact and $v \in W(K, B(x, 2 r))$. Then we have

$$
\int\left|\frac{\partial v\left(y^{\prime}, y_{n}\right)}{\partial y_{n}}\right| d y_{n} \geq 2 \quad \text { for all } y^{\prime} \in K .
$$

Hence it follows from Hölder's inequality and Fubini's theorem that

$$
\int|\nabla v|^{p} d \mu \geq c r^{-p+1+\alpha} \mathcal{H}^{n-1}(K)
$$

for a positive constant $c$. Consequently, we have

$$
\operatorname{cap}_{p, \mu}(F \cap B(x, r) ; B(x, 2 r)) \geq c r^{-p+1+\alpha} \mathcal{H}^{n-1}(F \cap B(x, r)) .
$$

In view of the assumption $\mathcal{H}^{n-1}(E)=0$ and Lemma 2.2, we see that

$$
\frac{\operatorname{cap}_{p, \mu}(F \cap B(x, r) ; B(x, 2 r))}{\operatorname{cap}_{p, \mu}(B(x, r) ; B(x, 2 r))} \geq c^{\prime}
$$

for a positive constant $c^{\prime}$. Now our claim is proved.

Fix $u \in W_{E}^{1, p}\left(\mathbf{R}^{n} ; \mu\right)$ such that $u$ is $(p, \mu)$-quasicontinuous on $\mathbf{R}^{n}$ and $\left.u\right|_{\mathbf{R}^{n-1}}=\chi_{E}$ on $\mathbf{R}^{n-1} \backslash A_{1}$, where $A_{1}$ has $(p, \mu)$-capacity zero. By Lemma 2.4, $u$ is $(p, \mu)$-finely continuous on $\mathbf{R}^{n} \backslash A_{2}$, where $A_{2}$ has $(p, \mu)$-capacity zero. Since $F$ is $(p, \mu)$-thick at each point of $E$ as was shown above,

$$
u=0 \quad \text { on } E \backslash A,
$$

where $A=A_{1} \cup A_{2}$. On the other hand, $u=1$ on $E \backslash A$, so that $E \backslash A$ is empty. Thus it follows that $\operatorname{cap}_{p, \mu}(E)=0$.

\section{$\S 5 . \quad$ Proof of Corollary $\mathbf{1 . 1}$}

Let $\nu$ denote the Borel measure on $\mathbf{R}^{n}$ defined by

$$
d \nu(y)=|1-| y||^{\alpha} d y .
$$

Proof of (1). Suppose that $w$ is a $(p, \nu)$-Dirichlet finite $(p, \nu)$-harmonic measure on B. Then $w(1-w) \in W^{1, p}(\mathbf{B} ; \nu)$ since $0 \leq w \leq 1$. By using $[3$, Theorem 3.17], there exists a solution $u \in W^{1, p}(\mathbf{B} ; \nu)$ of $(1.1)$ in $\mathbf{B}$ with $u-w(1-w) \in W_{0}^{1, p}(\mathbf{B} ; \nu)$. Since $0 \leq w \leq 1$, it follows from [3, Lemma 7.37] that $u$ is a $(p, \nu)$-harmonic minorant of both $w$ and $1-w$. By the definition of harmonic measures, we see that $u \leq 0$ in $\mathbf{B}$. Hence Theorem 1.1 implies that either $\operatorname{cap}_{p, \nu}(\{\zeta \in \partial \mathbf{B}: w(\zeta)=0\})=0$ or $\operatorname{cap}_{p, \nu}(\{\zeta \in \partial \mathbf{B}: w(\zeta)=1\})=$ 0 , so that $w$ is constant by $[3$, Lemma 7.37$]$. 
Proof of (2). Let $E$ be a domain in $\partial \mathbf{B}$ with (nonempty) smooth boundary and take $u \in W_{E}^{1, p}\left(\mathbf{R}^{n} ; \nu\right)$ as in Proposition 6.1 given in the next section. By $\left[3\right.$, Theorem 3.17] we find $w \in W^{1, p}(\mathbf{B} ; \nu)$ which is the solution of (1.1) in $\mathbf{B}$ with $w-u \in W_{0}^{1, p}(\mathbf{B} ; \nu)$. Clearly, $u$ and hence $w$ is not constant. Set $\hat{w}=\min \{w, 1-w\}$ and let $v$ be any $(p, \nu)$-harmonic minorant of $\hat{w}$. Since the boundary values of $w(1-w)$ are zero $(p, \nu)$-q.e, $\hat{w}$ has boundary values zero $(p, \nu)$-q.e. Hence [3, Lemma 7.37] implies that $v \leq 0$. It follows that $w$ is a $(p, \nu)$-harmonic measure on $\mathbf{B}$, which proves $(2)$.

\section{$\S 6$. Further results}

First we note the following result.

LEMma 6.1. If $0<\delta<1$ and $\Omega$ is a bounded domain with $C^{1}$ boundary, then

$$
\iint_{\Omega \times\left(\mathbf{R}^{n} \backslash \Omega\right)}|x-y|^{-n-\delta} d x d y<\infty .
$$

In fact, it suffices to note that

$$
\int_{\mathbf{R}^{n} \backslash \Omega}|x-y|^{-n-\delta} d y \leq \int_{\mathbf{R}^{n} \backslash B(x, d(x))}|x-y|^{-n-\delta} d y \leq c d(x)^{-\delta},
$$

for $x \in \Omega$,where $c$ is a positive constant and $d(x)=\operatorname{dist}(x, \partial \Omega)$ denotes the distance of $x$ from the boundary $\partial \Omega$.

Proposition 6.1. If $1<p<2+\alpha$ and $\Omega$ is a bounded domain on $\mathbf{R}^{n-1}$ with $C^{1}$ boundary, then $W_{\Omega}^{1, p}\left(\mathbf{R}^{n} ; \mu\right) \neq \emptyset$.

Proof. In view of Lemma 6.1, we see that $\chi_{\Omega}$ satisfies

$$
\iint_{\mathbf{R}^{n-1} \times \mathbf{R}^{n-1}} \frac{\left|\chi_{\Omega}\left(x^{\prime}\right)-\chi_{\Omega}\left(y^{\prime}\right)\right|^{p}}{\left|x^{\prime}-y^{\prime}\right|^{n+p-(2+\alpha)}} d x^{\prime} d y^{\prime}<\infty .
$$

Hence $\chi_{\Omega}$ belongs to the Lipschitz space $\Lambda_{\beta}^{p, p}\left(\mathbf{R}^{n-1}\right)$ with $\beta=1-(\alpha+1) / p$, so that the Poisson integral $u(x)=P_{x_{n}} * \chi_{\Omega}\left(x^{\prime}\right)$ satisfies

$$
\int_{\mathbf{D}}|\nabla u(x)|^{p} x_{n}^{\alpha} d x<\infty
$$

where $\mathbf{D}=\left\{x=\left(x^{\prime}, x_{n}\right): x_{n}>0\right\}$; see Stein [13] or the second author [9]. Then we can extend $u$ to a function in $W_{\Omega}^{1, p}\left(\mathbf{R}^{n} ; \mu\right)$, so that $W_{\Omega}^{1, p}\left(\mathbf{R}^{n} ; \mu\right)$ is not empty. 
For $K \subset \mathbf{R}^{n-1}$, let $\Lambda_{\beta}^{p, p}(K)$ be the space of all functions $u \in \Lambda_{\beta}^{p, p}\left(\mathbf{R}^{n-1}\right)$ such that $u=0$ outside $K$. Finally we discuss whether $\Lambda_{\beta}^{p, p}(K)$ with $\beta=$ $1-(\alpha+1) / p$ is trivial, or not. For this purpose we consider a capacity inequality introduced by Carleson [1, Theorem 2 in Section 4] (see also [8]).

Proposition 6.2. Let $K$ be a compact set in $\mathbf{R}^{n-1}$. Let $G_{1}$ and $G_{2}$ be bounded open sets in $\mathbf{R}^{n}$ such that $K \subset G_{1} \subset \overline{G_{1}} \subset G_{2}$. Set $\omega_{1}=$ $G_{1} \cap \mathbf{R}^{n-1}$. If $\beta=1-(1+\alpha) / p$ and

$$
\operatorname{cap}_{p, \mu}\left(\omega_{1} \backslash K ; G_{2}\right)<\operatorname{cap}_{p, \mu}\left(\omega_{1} ; G_{2}\right)
$$

then $\Lambda_{\beta}^{p, p}(K)$ has non-zero element.

Proof. We can find a $(p, \mu)$-quasicontinuous function $u_{1} \in W^{1, p}\left(\mathbf{R}^{n} ; \mu\right)$ such that

(i) $u_{1}=0(p, \mu)$-q.e. outside $G_{2}$;

(ii) $u_{1}=1(p, \mu)$-q.e. $\omega_{1} \backslash K$;

(iii) $\int_{G_{2}}\left|\nabla u_{1}\right|^{p} d \mu=\operatorname{cap}_{p, \mu}\left(\omega_{1} \backslash K ; G_{2}\right)$;

(iv) $\int_{G_{2}}\left|\nabla u_{1}\right|^{p-2} \nabla u_{1} \cdot \nabla \varphi d \mu=0$ for all $\varphi \in C_{c}^{\infty}\left(G_{2}\right)$ such that $\varphi=0$ on $\omega_{1} \backslash K$.

Similarly, we find a $(p, \mu)$-quasicontinuous function $u_{2} \in W^{1, p}\left(\mathbf{R}^{n} ; \mu\right)$ such that

(v) $u_{2}=0(p, \mu)$-q.e. outside $G_{2}$;

(vi) $u_{2}=1(p, \mu)$-q.e. $\omega_{1}$;

(vii) $\int_{G_{2}}\left|\nabla u_{2}\right|^{p} d \mu=\operatorname{cap}_{p, \mu}\left(\omega_{1} ; G_{2}\right)$;

(viii) $\int_{G_{2}}\left|\nabla u_{2}\right|^{p-2} \nabla u_{2} \cdot \nabla \varphi d \mu=0$ for all $\varphi \in C_{c}^{\infty}\left(G_{2}\right)$ such that $\varphi=0$ on $\omega_{1}$.

Then we see from (6.1) that $u_{1} \neq u_{2}$. Consequently, for $\varphi \in C_{c}^{\infty}\left(G_{1}\right)$, $\left.\left[\varphi\left(u_{2}-u_{1}\right)\right]\right|_{\mathbf{R}^{n-1}}$ is shown to be a non-zero element of $\Lambda_{\beta}^{p, p}(K)$. 


\section{REFERENCES}

[1] L. Carleson, Selected problems on exceptional sets, Van Nostrand, Princeton, 1967.

[2] L. C. Evans and R. F. Gariepy, Measure theory and fine properties of functions, Studies in Advanced Mathematics, CRC Press, 1992.

[3] J. Heinonen, T. Kilpeläinen and O. Martio, Nonlinear potential theory of degenerate elliptic equations, Oxford Univ. Press, 1993.

[4] D. A. Herron and P. Koskela, Continuity of Sobolev functions and Dirichlet finite harmonic measures, Potential Analysis, 6 (1997), 347-353.

[5] N. G. Meyers, A theory of capacities for potentials in Lebesgue classes, Math. Scand., 26 (1970), 255-292.

[6] Continuity properties of potentials, Duke Math. J., 42 (1975), 157-166.

[7] Y. Mizuta, Integral representations of Beppo Levi functions of higher order, Hiroshima Math. J., 4 (1974), 375-396.

[8] - On removable singularities of harmonic functions in $L^{p}$, Memoirs of the Faculty of Integrated Arts and Sciences, Hiroshima University, Ser. IV, Vol. 9 (1984) $9-21$.

[9] — Potential theory in Euclidean spaces, Gakkōtosyo, Tokyo, 1996.

[10] M. Nakai, Dirichlet finite harmonic measures on Riemann surfaces, Complex Variables, 17 (1991), 123-131.

[11] Existence of Dirichlet finite harmonic measures in non-linear potential theory, Complex Variables, 21 (1993), 107-114.

[12] — Existence of Dirichlet finite harmonic measures on the Euclidean balls, Nagoya Math. J., 133 (1994), 85-125.

[13] E. M. Stein, Singular integrals and differentiability properties of functions, Princeton Univ. Press, Princeton, 1970.

Toshihide Futamura

Department of Mathematics

Faculty of Science

Hiroshima University

Higashi-Hiroshima 739-8526, Japan

Yoshihiro Mizuta

The Division of Mathematical and Information Sciences

Faculty of Integrated Arts and Sciences

Hiroshima University

Higashi-Hiroshima 739-8521, Japan 